\title{
A FRAMEWORK FOR FACILITATING HIGHER- ORDER STRATEGIC THINKING IN ONLINE MANAGEMENT DEVELOPMENT
}

\author{
Paul Nicholson and Geoff White \\ Deakin University, $A U$
}

\begin{abstract}
Organizations which grow by responding dynamically and strategically to rapidly changing business environments require managers capable of analytical and creative thinking in individual and team contexts. Whilst management development of this kind is hard to achieve within a localised workplace, it is even more difficult in globally distributed workplaces. Appropriately designed online programs can facilitate effective management development within and across multi-site organizations. This requires, firstly, the insightful and purposeful development of a learning environment which is grounded in an understanding of the dynamics of learning and, secondly, the delivery of this environment online. These contexts require an instructional design model which focuses upon expertise development in collaborative settings - the key elements of our model.
\end{abstract}

Key words: management development, e-learning

\section{INTRODUCTION}

The majority of online instructional programs are transmissive, with an implied view of learning as passive rather than interactive and engaging attributes that are essential in environments designed for developing higherorder learning. Why do online course designers ignore such well-understood instructional approaches to the facilitation of effective learning? Presumably, this neglect is primarily a consequence of the focus of many such programs on developing lower-order thinking or skills emphasising the uptake of 
factual information or procedures. In this paper we argue that the dearth of explicit instructional models for developing higher-order thinking in online mode is a critical limiting factor in the development of quality online management development. As a step towards overcoming this barrier, we present a design model that we have developed and refined over the past ten years, and which we believe provides significant advantages for designing online learning environments for facilitating higher-order thinking.

\subsection{Why higher-order strategic thinking and learning?}

The culture of constant change which characterises contemporary workplaces is nowhere more apparent than in dynamic organisations which value and utilise their employees' capacities for strategic and responsive thinking. For such organizations, "learning is at the heart of productive activity - learning is the new form of labour." (Zuboff, 1988, p.395). The 'knowledge era' organisation generates its own knowledge and understandings and anticipates, defines and understands its own strategic opportunities. Such organisations place significant learning demands upon its employees as they operate within workplaces described by Barnett (1999) as characterised by 'supercomplexity'. Contrasting with these 'knowledge era' organisations are those that are simply reactive to change, preferring instead to focus upon the maintenance of traditional practices.

But what exactly is required of those who work in supercomplex contexts? Despres and Hiltrop (1995) identify the following attributes of a knowledge era workplace:

- knowledge and skills are specialised and deep;

- groups and projects provide the locus of activity;

- skill obsolescence is rapid;

- performance measures value process effectiveness;

- customers, problems and issues are the focus of work activity;

- employee loyalty is to the profession, networks and peers.

- the individual worker's impact on organisational performance is infrequent but strategic.

Within the context of online training and development, which design models will help people efficiently develop and refine their capacities to contribute to organisational survival in the new economies - to think and learn in higher-order ways? The cognitive competencies involved in such thinking include the ability to: 
- analyse claims, opinions and complex situations;

- make critical judgements of alternative views;

- evaluate and synthesise a variety of positions into a coherent whole;

- apply their knowledge and understanding in productive and defensible ways;

- meet strategic challenges facing their organisation.

Before elaborating our design model, we will outline the distinction between active and passive learning and the attributes of the former that underpins the model.

\section{ONLINE ENVIRONMENTS FOR ACTIVE LEARNING AND HIGHER-ORDER THINKING.}

In this paper, we contrast traditional education and training contexts with those designed to facilitate active learning and higher-order thinking. Traditional contexts focus on the development of clearly defined workplace knowledge and skills, and constitute a bounded knowledge system (Nicholson \& White, 2001; Nicholson, 1999). These usually require only the incisive and expert transfer and application of existing knowledge to new, but predictable, situations. In such cases, there is a well-defined knowledge base of content, rules, and practices that can be used to guide the development of both traditional and online training programs (e.g., Murray, 1996), the majority of which focus on developing low-level skills and knowledge.

In contrast, when the focus of 'instruction' shifts from simply delivering content to the more complex development of higher-order thinking, naive assumptions about the passive learning of content have to be replaced by a more sophisticated understanding of the requirements of person-centred active-learning models. This reconceptualisation is necessary because "Higher-order thinking occurs when a person takes new information and information stored in memory and interrelates and/or rearranges and extends this information to achieve a purpose or find possible answers in perplexing situations." (Lewis \& Smith, 1993). Learning of this kind is, therefore, cognitively active learning.

Cognitive complexity is the hallmark of such learning contexts and requires an active learning sequence (Figure 1) for the achievement of the necessary higher-order learning. Initial exploration and engagement is a key element of an active learning sequence, as this engages and contextualises issues for the learners by giving them with the opportunity to locate the issue 
or content in their own workplace context. Contextualisation requires learners to bring their prior knowledge, experiences, and interpretations into an authentic learning sequence (DeMartino, 1999, p.786).

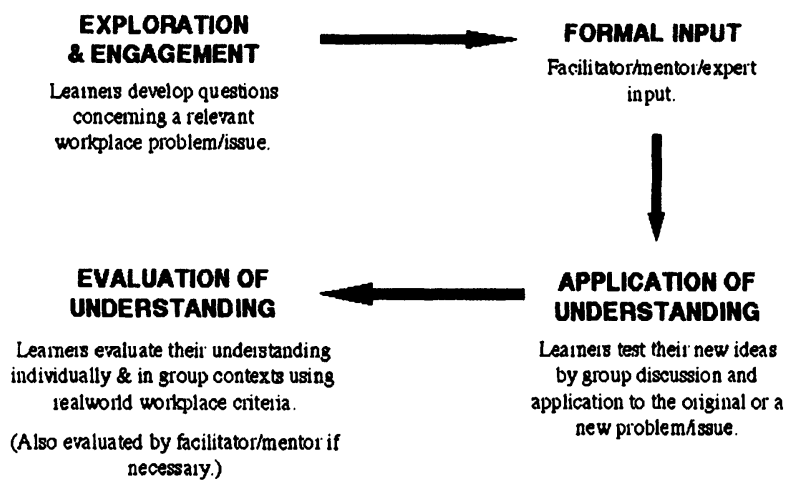

Figure1. An active learning sequence (Nicholson \& White, 2000)

Formal instructor/mentor/facilitator input into the sequence is also required, as simply engaging with the issues does not guarantee higher-order learning will occur. This stage, of course, is one that totally dominates many passive learning models. Subsequent stages in the active learning sequence have the learners testing their own understandings by applying them to new problems and, in some cases, evaluating their own understandings. This combination of a focus on higher-order thinking, active learning, and a situative approach has been described as a 'Rich Environment for Active Learning' (REAL) (e.g., Dunlap, 1999).

\section{ACTIVE LEARNING ONLINE}

Figure 2 depicts a conceptual design for an online REAL. 


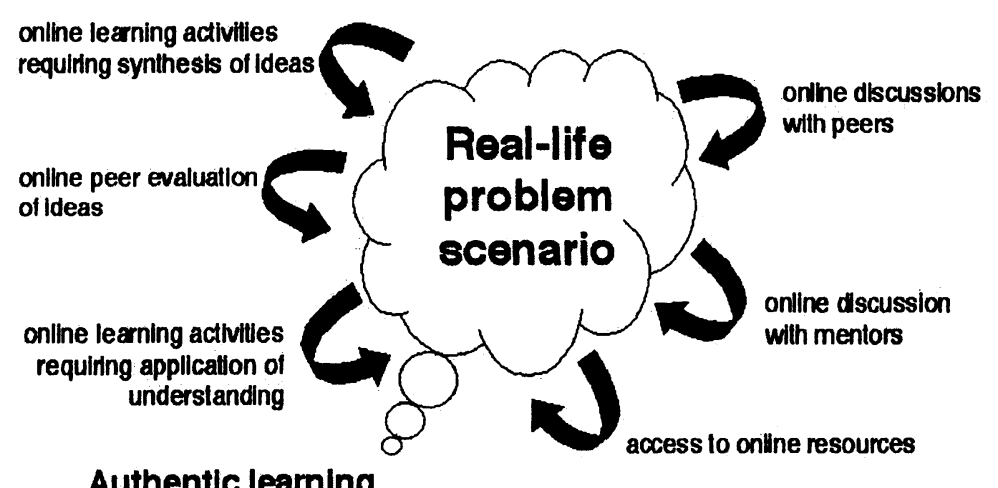

Figure 2. Conceptual design of a generic online REAL. (Nicholson \& White, 2000)

The critical aspect of a REAL is the engagement and interaction of participants between themselves, external mentors, and the course content. Online technologies are able to support such interaction, and can be used to structure interactions amongst the participants of a REAL. The design is based on our work over the past 10 years in developing online adult learning environments for facilitating higher-order thinking. It represents, in general terms, the operationalisation of the REAL model in an online format.

\section{FUZZY CONTEXTS AND HIGHER-ORDER THINKING}

The hypercomplex nature of most contemporary business environments means there is no well-articulated (or perhaps cohesive) knowledge base, clear solution, best strategy, or readily available basis for decision-making. Given that such unbounded knowledge systems lack ready-made answers to be 'learned', they require a 'fuzzy' learning design that accommodates uncertainty as a key design feature, with a focus on higher-order thinking. In fuzzy designs, the learner has to actively develop an answer meaningful within the context of the learning experiences provided by the learning facilitator or course. Examples of contemporary fuzzy problems in a 'realworld' business context include issues such as deciding on strategic ecommerce structures and processes; meeting the challenges of globalisation and long-term strategic investment policy in unstable markets. Such fuzzy problems are not generally amenable to the simple solutions found in predesigned course materials. Designers trying to develop courses for such fuzzy contexts have to address a set of difficult questions that include:

1. How are higher-order learning $\&$ knowledge growth facilitated? 
2. How are tasks with 'fuzzy' outcomes and unbounded knowledge bases structured and sequenced?

3. How can existing expertise and 'intellectual capital' be best utilised?

4. How are 'fuzzy' program outcomes evaluated?

In general, traditional instructional design paradigms are unable to cope with the nature, scope, and incorporation of uncertainty and values that fuzzy contexts contain. Based on our experiences, and our analysis of the weakness of many instructional design paradigms (in this context), we argue that any instructional design model that attempts to address fuzzy contexts must replace the traditional instructional design process of creating a predetermined learning sequence with one that creates a set of loosely structured learning pathways, as the notion of sequential learning is problematic in fuzzy contexts. There is a clear need for a range of new design paradigms that can be adapted to suit a wide range of fuzzy contexts. The layered-design model below is one such approach to developing higherorder thinking in fuzzy contexts that has proven its value in this regard.

\section{A MODEL FOR FUZZY ENVIRONMENTS}

The layered-design model (Figure 3) was developed by us over time, as it became clear that in designing for fuzzy contexts, at least four separate design foci had to be considered.

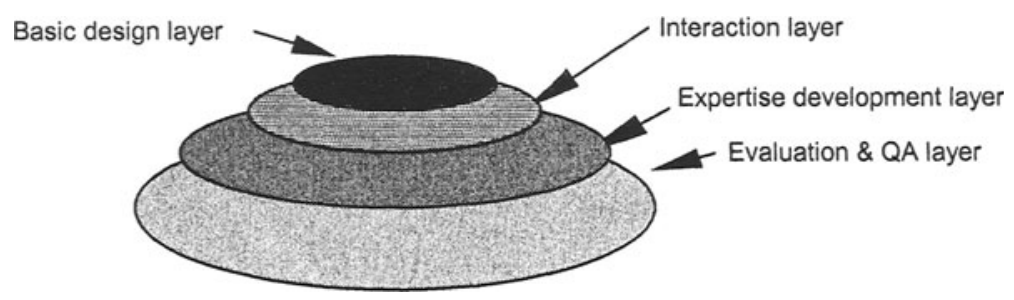

Figure 3: A layered-design model for fuzzy environments (Nicholson \& White, 2001)

These foci are (a) technical aspects; (b) how and why learners will interact with the course content, other participants, and external individuals; (c) the instructional structures and processes that build upon the interactions to develop expertise in the particular fuzzy context; (d) the authentic evaluation/assessment processes that need to be developed to ensure the attainment of expertise can be evaluated in a meaningful manner. While these may appear to be straightforward, in our experience it is not an easy 
task to develop the content and processes with the same level of certainty as in a traditional design context. Indeed, the design task becomes more of a 'crafting' of the learning environment than one of technical construction.

The first, and perhaps easiest, design task is addressed in the basic design layer - the choice of platform, software, etc. These considerations, often seen as precursors to the design process, have to be integrated developmentally because of the implications of such decisions for the rest of the design process. In other words, learning needs, not the platform or software, should dictate the design (cf. Alexander, 1999).

Figure 3 is an abstraction Figure 2, focusing on ensuring that specific aspects of the online discussions and engagement with content and resources are properly structured to achieve the desired learning outcomes. In this model, content, while critical, is no more important than the discussions and arguments associated with its analysis. We have found it is important to differentiate between interaction and the development of expertise as the two are fundamentally distinct, a point often overlooked in traditional contexts. Simply planning structures and processes such as learning sequences, discussions and online seminars, etc. to engage learners with content is insufficient for the facilitation of higher-order learning. These components must be supplemented by the purposeful development of expertise arising from such interactions.

The situative nature of fuzzy contexts has implications for the expertise development stage. For example, in the case of a business trying to address the challenges of globalisation, the required expertise is not only that of understanding the issues, but also includes an understanding of situative aspects such as strategies for the development of a management-team approach, the sharing of understandings and the leveraging of group intellectual capital in the pursuit of corporate goals. The desired outcomes include personal and group (situative) aspects that have to be developed along with whatever content knowledge is needed to support them. Online conferences are critical facilitators of this social interaction.

The evaluation process is also affected by the same kinds of issues. The notion of peer and self-assessment was raised in Figure 2, and it is highly relevant here. For example, how should the evaluation of the business case (above) be conducted, and by whom? There is clearly no 'correct' answer to assess, other than that the participants' ability to perform the desired tasks has improved, an issue best determined in the workplace. In such cases, the assessment layer design would include a significant element of employer evaluation of performance over time, a distinguishing feature relative to the all too common 'end of course' competency assessment which is axiomatically unsuited to fuzzy contexts. 


\section{CONCLUSION}

Grounded in notions of active learning and authentic contextual settings, the design model outlined in this paper, in conjunction with the active learning model (Fig.2), stands in stark contrast to many of the masterylearning based models currently used for knowledge and procedural training. Our model has been developed for, and implemented within, a range of higher-order learning purposes and through this experience we have learned the significant dependence of the model upon detailed knowledge of the learning theories literature, an understanding of the dynamics of learning, and expertise in the delivery of instruction designed to facilitate higher-order thinking and learning.

Our experience also affirms the importance of these understandings and competencies, rather than technical imperatives, as the primary driver of design for higher-order learning online. After all, online technologies are a medium, not a training solution, most particularly so when the desired training outcome is the higher-order strategic thinking valued by today's knowledge era organisations.

For organisations seeking to leverage the intellectual capital of its managers, higher-order thinking must be the primary focus of its development activities. This paper proposes that the utilisation of the online medium for this purpose is optimally served by the model outlined above. Our experience with this model in a variety of contexts leads us to believe it has significant power to achieve the types of thinking and learning required of managers in dynamic and responsive growth organisations, most particularly those with a distributed workforce.

\section{REFERENCES}

Alexander, S. (1999). Selection \& evaluation of the TopClass WWW-based course support tool. International Journal of Educational Telecommunications, 5(4), 271-283

Barnett, R. (1999). Learning to work and working to learn. In D. G. Boud, J. (Ed.), Understanding learning at work. London: Routledge

DeMartino, D. J. (1999). Employing adult education principles in instructional design. In J. D. Price \& J. Willis (Eds.), Proceedings of SITE 999, San Antonio, Texas (pp. 783-788). Charlottesville, VA: AACE

Despres, C. H., \& Hiltrop, J.M. (1995). Human resource management in the knowledge age: current practice and perspectives on the future. Employee Relations, 17(1), 9-23

Dunlap, J. (1999). Rich environments for active learning on the Web: Guidelines and Examples. In P. de Bra \& J. Leggett (Eds.), Proceedings of WebNet 99. Honolulu, Hawaii. Charlottesville, VA: AACE

Lewis, A., \& Smith, D.(1993). Defining higher-order thinking. Theory into Practice,32(3),131 
Murray, T. (1996). Special purpose ontologies and the representation of knowledge. In D. C. Edelson \& E. A. Domeshek (Eds.), Proceedings of ICLS96, Northwestern University July 25-27 (pp. 235-242). Evanston. IL

Nicholson, P. S. (1999). Linking science thinking and learning with software. Paper presented at COMNED'99: Networking the learner., Haamelina, FI

Nicholson, P.S., \& White, G.D. (2001) Teaching for quality learning online: A layered Design model for higher-order thinking. in D.Watson \& J.Andersen (Eds.)., Computers and Education. New York: Kluwer (in press).

Nicholson, P. S., \& White, G. D. (2000, September 5th). Rich environments for active learning. Paper presented at the AITD seminar on Adult Learning, Melbourne

Zuboff, S. (1988). In the age of the smart machine: the future of work and power. New York: Basic Books.

\section{BIOGRAPHY}

Paul Nicholson and Geoff White have research interests in the design and use of online environments for developing higher-order thinking and the development of ICT-based tools to support effective online teaching.. 